\title{
Safety and Tolerability of a 13-Valent Pneumococcal Conjugated Vaccine Distributed in the Public Immunization Program of the Municipality of Campos dos Goytacazes, Rio de Janeiro, Brazil
}

\author{
Charbell Miguel Kury ${ }^{1,2 *}$, Annelise Maria Wilken ${ }^{1,2}$, Raquel Mesquita Henriques², Renata de Salles², \\ Wilza Abreu de Brito ${ }^{2}$, Marcus Miguel Kury ${ }^{1}$, Rafael Pessanha de Sá ${ }^{2}$, Felipe Pessanha de Sá ${ }^{2}$ \\ ${ }^{1}$ Secretariat of Health, Municipality of Campos dos Goytacazes, State of Rio de Janeiro, Brazil; ${ }^{2}$ Medicine School of the Munici- \\ pality of Campos dos Goytacazes, State of Rio de Janeiro, Brazil. \\ Email: *charbellkury@hotmail.com
}

Received: September $13^{\text {th }}, 2012$; revised October $25^{\text {th }}, 2012$; accepted November $5^{\text {th }}, 2012$

\begin{abstract}
From september to december, 2010, we have assessed the frequency and occurrence of adverse events to Pneumococcal conjugated 13-valent vaccine (PCV-13) in the Public vaccination program of the municipality of Campos dos Goytacazes, State of Rio de Janeiro, the unique city in Brazil that has introduced this vaccine in it's immunization schedule. This study analyzed 1001 toddlers who have received PCV-13 at 3,5 and 7 months and a booster dose at 12 months. We observed a total of 514 local and systemic events in 303 subjects (30.2\% of 1001 infants). The most reported systemic events were irritability $(18.8 \%)$ and fever $<38.5^{\circ} \mathrm{C}(17.1 \%)$, followed by fever $>$ or $=38.5^{\circ} \mathrm{C}(8.8 \%)$, loss of appetite $(8.4 \%)$. Erythema $(11.2 \%)$ and local pain $(9.4 \%)$ were the most reported local events. Other events reported were diarrhea (6.2\%), increased sleep (5.1\%), edema and induration (4.8\%), decreased sleep (4.3\%), vomiting (1.4\%), eruption $(1.2 \%)$ urticaria $(0.8 \%)$, prurience $(0.8 \%)$, lymphadenopathy $(0.2 \%)$ and hypersensitivity reaction $(0.2 \%)$. There wasn't any reported case of convulsion or Hospital admission. When stratified by each dose, irritability (systemic) and erythema (local) were the most common events reported at the first and fourth dose, although fever $<38.5^{\circ} \mathrm{C}$ (systemic) and pain (local) were the most common at second and third doses. Results were close to those encountered in product monograph. In our study, PCV-13 was secure in pneumococcal disease prevention and well tolerated.
\end{abstract}

Keywords: Streptococcus pneumoniae; PCV-13, Adverse Events; Pneumococcal Disease

\section{Introduction}

Streptococcus pneumoniae is usually carried in the nasopharynx of healthy people, but occasionally leads to pneumococcal diseases (PD), such as meningitis, pneumonia, otitis, sinusitis and bacteremia. Annually, World Health Organization (WHO) estimates the occurrence of one and a half million of deaths in children under five years by PD, mainly in poor countries [1,2]. In Brazil, the age group that accounts for the major rates of morbidity and mortality by PD is the group of children under two years old, mainly by pneumococcal meningitis. Brazilian data sustain that this condition leads to lethality rate of $46 \%$ and $50 \%$ of rate for neurological disorders and hearing sequelae $[3,4]$. After introduction of Pneumoccocal Conjugated 7-valent vaccine (PCV-7) in USA children immunization schedule, in 2000 [5], invasive

${ }^{*}$ Corresponding author.
PD has decreased $76 \%$. Nevertheless, in 2002 it was observed an increase in rate of invasive PD incidence due to non PCV-7 serotypes, mainly serotype 19A, in which incidence levels have increased more than 200\% [6-8]. Important consideration about serotype 19 is related to the increased Pneumococcal resistance to antimicrobials, which had motivated the development of newer pneumococcal conjugated vaccines, like the pneumococcal conjugated 10 and 13-valent (PCV-13) [9].

PCV-13 was introduced in public vaccination program of the municipality of Campos dos Goytacazes, Brazil, in September, 2010, replacing PCV-7, which was introduced in this municipality in May 29, 2009, and has been the first and the unique city that has introduced PCV-7 and PCV-13 in a public program of immunization in Brazil. The PCV-13 had been chosen because the possibility to replacement of PCV-7 to PCV-13 in the infant schedule in any moment of vaccination $[10,11]$. 
This study aims to assess the frequency and occurrence of adverse events to PCV-13 in the Municipality of Campos dos Goytacazes, by using active surveillance. That's the first study in Brazil, since there's no publication in this country related to adverse events to PCV-13 in a public Program of Immunization.

\section{Methods}

Campos dos Goytacazes, the largest municipality in State of Rio de Janeiro, Brazil has $4.032 \mathrm{Km}^{2}$ of territorial distribution. Population is about 463,545 individuals, according to Government 2010 official census, and the economy is supported by petroleum extract, which represents $80 \%$ of the Brazilian production.

PCV-13 was licensed in February 24, 2010 by Food and Drug Administration (FDA) and has been approved and recommended by the Advised Committee on Immunization Practice (ACIP) to prevent invasive PD and acute otitis media in children [5]. PCV-13 comprises 13 S. pneumoniae polysaccharides serotypes $1,3,4,5,6 \mathrm{~A}$, $6 \mathrm{~B}, 7 \mathrm{~F}, 9 \mathrm{~V}, 14,18 \mathrm{C}, 19 \mathrm{~A}, 19 \mathrm{~F}$ e $23 \mathrm{~F}$. It also contains 2.2 $\mu \mathrm{g}$ of each saccharide, except for $4.4 \mu \mathrm{g}$ of serotype $6 \mathrm{~B}$. This vaccine also contains $32 \mu \mathrm{g}$ of $\mathrm{CRM}_{197}$, a nontoxic variant of diphtheria toxin. The adjuvant used in PCV-13 was $0.125 \mathrm{mg}$ of aluminum phosphate in $0.5 \mathrm{ml}$-dose. The PCV-13 schedule used in Campos dos Goytacazes are three doses in the primary vaccination series, with first dose at 3 months, second dose at 5 months, and third dose at 7 months, followed by a booster dose at 12 months $[5,10,11]$.

PCV-13 adverse events in the product monograph are classified in: Very Common (>10\%): decreased appetite, irritability, increased sleep, decreased sleep, fever $<39^{\circ} \mathrm{C}$, tenderness, erythema, local edema; Common $(>1 \%$ e $<$ $10 \%$ ): diarrhea, vomiting, eruption, fever $>39^{\circ} \mathrm{C}$; Less Common $(>0.1 \% \mathrm{e}<1 \%)$ : seizures, including febrile seizures, urticaria, local induration/erythema or induration $>$ $7.0 \mathrm{~cm}$; Rare $(>0.01 \%$ e $<0.1 \%)$ : Hypersensitivity reaction, dyspnea, bronchospasm; e Very rare $(<0.01 \%)$ : Lymphadenopathy, anaphylactic reaction, multiform erythema [11].

There were enrolled in the observational and descriptive study 1.001 healthy toddlers that had received the PCV-13 from September 2010 to december 2010 at the vaccine room located at the Secretariat of health of the Municipality of Campos dos Goytacazes. Inclusion criteria included infants who are residents in the municipality and without any disease. Exclusion criteria included patients with comorbidities.

Sample size calculation have used the OPENEPI ${ }^{\circledR}$ tool, and the variables involved were 8000 individuals, related to the people under 2 years old in the municipality, considering a confidence limit of $5 \%$ and power test of
99.99\%. Sample was calculated using the estimated frequency of $30 \%$ of adverse events [12].

In study period, infant parents that were elected for the research filled written informed consent, and personal data were asked. In the toddler vaccine record was attached a form, containing adverse events list based in product monograph. Parents were oriented to observe the occurrence of adverse events in 48 hours and fill in the events form. After 48 hours, parents received a phone call from the research staff to ask for the occurrence of any event.

Data were inserted in EpiData ${ }^{\circledR}$ version 3.1, and was used to build the data bank. Then, there was used EpiAnalysis ${ }^{\circledR}$ program, to the arrange data bank and analysis. Charts and tables were build in Microsoft ${ }^{\circledR}$ Office Word 2010 and Adobe Photoshop ${ }^{\circledR}$.

Our study was approved in the research ethics committee of the Medicine School of Campos dos Goytacazes, Rio de Janeiro, Brazil.

\section{Results}

Table 1 summarizes the sample in general features. There was a predominance of the male gender, with 527 infants $(52.6 \%)$. The mainly age group was the group between 2 and 18 months of age (81\%). Nevertheless, first dose of the primary series was the most represented in the study (32.3\%).

Occurrence of adverse events was observed in 303 infants $(30.2 \%)$, who had related at least one event, accor-

Table 1. Subject Demographic characteristics that received PCV-13 in Campos dos Goytacazes, Brazil.

\begin{tabular}{cc}
\hline Variables & N (\%) \\
\hline Sample Vaccinated & $1001(100)$ \\
Female & \\
Male & $474(47.4)$ \\
Age Group Vaccinated & $527(52.6)$ \\
$02-12$ months & \\
$13-24$ months & $186(18.6)$ \\
$>24$ months & $4(0.4)$ \\
Dose Received & \\
First & $323(32.3)$ \\
Second & $272(27.2)$ \\
Third & $212(21.2)$ \\
Fourth (Booster) & $194(19.3)$ \\
\hline
\end{tabular}


ding to Table 2. In this group that reported adverse events was observed that there wasn't any significant difference in male $(52.8 \%)$ or female $(47.1 \%)$ gender. When stratified by dose received, the most reported events were related to the primary vaccination series, mainly first and second doses, and represented $73 \%$ of total of events reported. However, booster dose was the less representative of the total of adverse events related, with $8.6 \%$.

Other variable observed was the percentage of infants that reported only one or multiple events. Investigation concluded that $49.5 \%$ of toddlers reported one event and $50.5 \%$ multiple events.

Table 3 summarizes the entire group adverse events. In 303 infants were observed 514 events. Erythema and Pain were the local events most reported, with $11.2 \mathrm{e}$ $9.3 \%$, respectively. Irritability and fever less than $38.5^{\circ} \mathrm{C}$ was the systemic events most reported, with $18.8 \mathrm{e}$ $17.1 \%$ of total of events related, respectively.

Graphics 1 and 2 summarize the occurrence of local and systemic events by each dose received. Toddlers that received first and fourth doses, the most reported systemic events were irritability and fever less than $38.5^{\circ} \mathrm{C}$. On the other hand, toddlers who had received second and third dose, fever less than $38.5^{\circ} \mathrm{C}$ and irritability was the most reported, respectively. When stratified for local events, erythema was the most reported in all doses,

Table 2. Proportions of subjects that reported adverse events.

\begin{tabular}{cc}
\hline Variables & N (\%) \\
\hline Sample Vaccinated & $1001(100)$ \\
Adverse Events & $303(30.2)$ \\
GENDER & \\
Female & $143(47.1)$ \\
Male & $160(52.8)$ \\
Adverse Events By Dose & \\
Received & $124(40.9)$ \\
First & $97(32.0)$ \\
Second & $56(18.5)$ \\
Third & $26(8.6)$ \\
Fourth (Booster) & \\
Reporting of the Event & $150(49.5)$ \\
By Single Event & $153(50.5)$ \\
By Multiple Event &
\end{tabular}

mainly in first one. Less often, edema and local induration had the same results at the first 3 doses, except fourth dose.

Table 3. Total of participants that reported adverse events and proportions of local and systemic events. Campos dos Goytacazes, Brazil.

\begin{tabular}{|c|c|}
\hline Local and Systemic Events & $514(100 \%)$ \\
\hline \multicolumn{2}{|l|}{ Local } \\
\hline Erythema & $58(11.2)$ \\
\hline Pain & $48(9.3)$ \\
\hline Edema and Local Induration & $25(4.8)$ \\
\hline Eruption & $6(1.2)$ \\
\hline Prurience & $4(0.8)$ \\
\hline \multicolumn{2}{|l|}{ Systemic } \\
\hline Fever & $141(27.4)$ \\
\hline$>$ or $=38.5^{\circ} \mathrm{C}$ & $45(8.8)$ \\
\hline$<$ or $=38.5^{\circ} \mathrm{C}$ & $96(17.1)$ \\
\hline Irritability & $97(18.8)$ \\
\hline Loss of appetite & $43(8.4)$ \\
\hline Diarrhea & $32(6.2)$ \\
\hline Increased Sleep & $26(5.1)$ \\
\hline Decreased Sleep & $22(4.3)$ \\
\hline Vomiting & $7(1.4)$ \\
\hline Urticaria & $4(0.8)$ \\
\hline Hypersensitivity reaction & $1(0.2)$ \\
\hline Lymphadenopathy & $1(0.2)$ \\
\hline Convulsion & $0(0)$ \\
\hline
\end{tabular}

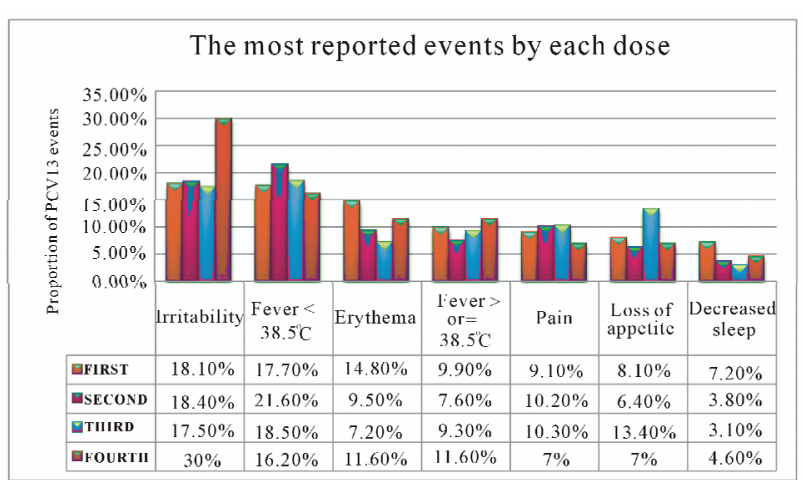

Graphic 1. The most reported adverse events to PCV-13 by each dose. 


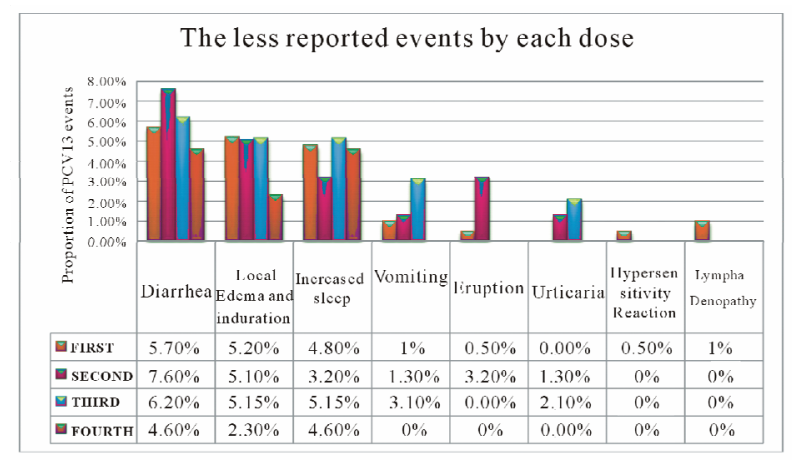

Graphic 2. The less reported events by each dose.

There was related only one hypersensitivity reaction in first dose, however, there wasn't classified in Serious adverse events (SAE) and didn't have hospital admission. There was also reported one case of lymphadenopathy after vaccination.

\section{Discussion}

Our study is the first one in a Public Program of Immunization in Brazil that was designed to assess the occurrence and frequency of adverse events to PCV-13 using active surveillance. In Brazil, PCV-13 is only sold in vaccination Private Clinics, except the municipality of Campos dos Goytacazes, State of Rio de Janeiro, who had started public vaccination with PCV-13 to infants less than 2 years old. Important outcomes in reducing the incidence of pneumococcal diseases have been encountered after administration of at least 40,000 doses of PCV-7 and PCV-13 vaccines in more than 10,000 toddlers. Data of the National System of Notification (SINAN) of Brazilian Minister of Healthy considered that the municipality of Campos dos Goytacazes have decreased $100 \%$ of the incidence of pneumococcal meningitis in 2010, one year after the introduction of PCV-7, in all age group. This data suggests the possibility of herd immunity influence, since the vaccination coverage for PCV-7 and PC-13 have already crossed the line of 95\%.

Firstly, there may be a parallel between the results of our study and that encountered in product monograph [11]. In this way, the events listed like very common in monograph, our study related irritability and fever less than $38.5^{\circ} \mathrm{C}$, except for erythema, edema and local induration, classified like common events. Other events, such as diarrhea and vomiting were considered common events, according to the monograph. The same found occurred in less common events, such as urticaria, unless rare events like hypersensitivity reaction and very rare like lymphadenopathy had occurred more in our study than in monograph.

Secondly, There also may be a comparison with this study and that of Bryant and cols. [13], which have randomly selected 122 toddlers who have received PCV-13 and 127 infants that have received PCV-7. After 15 days, there was observed the same outcome encountered in systemic events at first and third doses. In decreasing order, there was found irritability, increased sleep, decreased appetite, decreased sleep and fever less than $39^{\circ} \mathrm{C}$. For the second dose, decreased sleep and decreased appetite change their frequency. In the other hand, edema/ local induration and erythema were the most frequent events, in decreasing order.

The revision article developed by Adis Drug Profile in 2010 assessed data from a meta-analysis with 12 clinical trials for 4429 infants, which had verified safety and tolerability of PCV-13. Local pain was the most observed local event, and irritability, the systemic event most reported. Nevertheless, our study has reported that irritability and erythema was the most related systemic and local events, respectively. In meta-analysis there were found other proportions of local and systemic events not related in frequency with our study. In decreasing order, local events list is followed by erythema and edema/local induration, while in systemic events were also encountered sleep disorders, decreased appetite and fever. In our study there's a change in this order: in local events, erythema is followed by pain and edema; in systemic events, irritability is followed by fever, decreased appetite and sleep disorders [14].

In other related article Snape and cols. [15] had analyzed 141 toddlers who had received PCV-13 and 145 infants which had received PCV-7, randomly selected. Results were similar to that from Bryant and cols. [13], in which the most common systemic events were irritability, with more that $40 \%$ frequency in any of the four doses. About local events, most common was edema and local induration, followed by erythema, with more than $20 \%$ frequency.

In recent study, Vanderkooi e cols. [16] randomly selected 300 toddlers who had received PCV-13 and 303 had received PCV-7. In this study, irritability was the most common adverse event, followed by fever less than $39^{\circ} \mathrm{C}$. When stratified by local events, edema/local induration and erythema were the most common, respectively.

There are several potential limitations in our study. In a general view, although this research has reached percentage of events close to that encountered at PCV-13 monograph, when compared to other international studies the events were less frequent. There may be considered that the other studies analyzed have observed the onset of adverse events for a longer period of time, ranging from 4 to 15 days, while our study have observed for 48 hours. Furthermore, this percentage could be affected by the 
sample size enrolled; demographic, social and cultural factors, and vaccine lot composition.

Overall, this observational and descriptive study have demonstrated the safety and tolerability of PCV-13 in the municipality of Campos dos Goytacazes-RJ, evidenced by low percentage of adverse events observed through active surveillance. Furthermore, the absence of SAE, and no need for hospital admission, added to the benefits in reducing incidence of pneumococcal meningitidis after begining PCV-7 and PCV-13 in this municipality have ensured the magnitude of benefits of PCV-13.

\section{Acknowledgements}

We acknowledge Prof. MD. Luiz Claudio Teixeira, Epidemiologist, for his revision in data.

\section{REFERENCES}

[1] World Health Organization, "Pneumococcal Conjugate Vaccine for Childhood Immunization," 2007. http://www.who.int/wer/2007/wer8212

[2] L. Ferro, et al., "Pneumococcal Conjugate Vaccine Impact against Invasive Disease Caused by Streptococcus pneumoniae," Brazilian Journal of Pediatrics, Vol. 82, No. 3, 2006, pp. S67-S74.

[3] G. Vespa, D. O. Constela, C. Pepe, M. A. Safadi, E. Berezin, et al., "Estimating the Cost Effectiveness of Pneumococcal Conjugate Vaccination in Brazil," Pan American Journal of Public Health, Vol. 26, No. 6, 2009, pp. 518-28. doi:10.1590/S1020-49892009001200007

[4] D. Constela, "Evaluating the Costs of Pneumococcal Disease in Selected Latin American Coutries," Revista Panamericana de Salud Pública, Vol. 22, No. 4, 2007, pp. 268-278.

[5] Center of Disease Control and Prevention, "Invasive Pneumococcal Disease in Young Children before Licensure of 13-Valent Pneumococcal Conjugate VaccineUnited States, 2007," Morbity and Mortality Weekly Report, Vol. 59, No. 9, 2010, pp. 253-257.

[6] S. I. Pelton, H. Huot, J. A. Finkelstein, et al., "Emergence of 19A as Virulent and Multidrug Resistant Pneumococcus in Massachusets Following Universal Immunization of Infants with Pneumococcal Conjugate Vaccine," The Pediatric Infectious Disease Journal, Vol. 26, No. 6, 2007, pp. 468-472. doi:10.1097/INF.0b013e31803df9ca
[7] Centers for Disease Control and Prevention, "Emergence of antimicrobial-resistant serotype 19A Streptococcus pneumoniae-Massachusetts, 2001-2006," Morbity and Mortality Weekly Report, Vol. 56, No. 41, 2007, pp. 1077-1080.

[8] T. Pilishvili, C. Lexau, M. M. Farley, et al., "Sustained Reductions in Invasive Pneumococcal Disease in the Era of Conjugate Vaccine," The Journal of Infectious Diseases, Vol. 201, No. 1, 2010, pp. 32-41. doi:10.1086/648593

[9] E. C. Dinleyici and Z. A. Yargic, "Current Knowledge Regarding the Investigational 13-Valent Pneumococcal conjugate Vaccine," Expert Review of Vaccines, Vol. 8, No. 8, 2009, pp. 977-986. doi:10.1586/erv.09.68

[10] Food and Drug Administration (FDA), "Vaccines: Approved Products. Prevnar 13 (Pneumococcal 13-Valent Conjugate Vaccine)," 2010.

http://www.fda.gov/biologicsbloodvaccines/vaccines/appr ovedproducts/ucm201667.htm

[11] Pfizer $^{\circledR}$ and Wyeth ${ }^{\circledR}$. "Pneumococcal 13-Valent Conjugate Vaccine Monography Product (Prevenar 13)," Brazilian Ministery of Healthy, 2010.

[12] A. G. Dean and K. M. Sullivan, "OpenEpi Collection of Epidemiologic Calculators," Version 2.3, 2009. http://www.openepi.com/OE2.3/Menu/OpenEpiMenu.ht $\mathrm{m}$

[13] K. A. Bryant, S. L. Block, S. A. Baker, et al., "Safety and Immunogenicity of a 13-Valent Pneumococcal Conjugate Vaccine," Pediatrics, Vol. 125, No. 5, 2010, pp. 866-875.

[14] S. T. Duggan, "Pneumococcal Polysaccharide conjugate vaccine (13-Valent, Adsorbed) [PREVENAR 13 $\left.{ }^{\circledR}\right]$," Drugs, Vol. 70, No. 15, 2010, pp. 1973-1986. doi:10.2165/11205110-000000000-00000

[15] M. D. Snape, C. L. Klinger, E. D. Daniels, et al., "Immunogenicity and Reactogenicity of a 13-Valent Pneumococcal Conjugate Vaccine Administered at 2, 4 and 12 Months of Age," The Pediatric Infectious Disease Journal, Vol. 29, No. 12, 2010, pp. e80-e90. doi:10.1097/INF.0b013e3181 faa6be

[16] O. G. Vanderkooi, D. W. Scheifele, D. Girgenti, et al., "Safety and Immunogenicity of a 13-Valent Pneumococcal Conjugate Vaccine in Healthy Infants and Toddlers Given with Routine Pediatric Vaccinations in Canada," The Pediatric Infectious Disease Journal, Vol. 31, No. 1, 2012, pp. 72-77. doi:10.1097/INF.0b013e318233049d 